\title{
Thermophysical properties of the lanthanide sesquisulfides. II. Schottky contributions and magnetic and electronic properties of $\gamma$-phase $\operatorname{Pr}_{2} \mathrm{~S}_{3}$, $\mathrm{Tb}_{2} \mathrm{~S}_{3}$, and $\mathrm{Dy}_{2} \mathrm{~S}_{3}$
}

\author{
John B. Gruber \\ Department of Physics, San Jose State University, San Jose, California 95192 \\ Ramón Burriel \\ Instituto de Ciencia de Materiales de Aragón, Universidad de Zaragoza-CSIC, 50009 Zaragoza, Spain \\ Edgar F. Westrum, Jr., W. Plautz, G. Metz, and Xiao-Xia Ma \\ Department of Chemistry, University of Michigan, Ann Arbor, Michigan 48109 \\ B. J. Beaudry and P. E. Palmer \\ Ames Laboratory, Iowa State University, Ames, Iowa 50011
}

(Received 20 November 1990; accepted 16 April 1991)

\begin{abstract}
Heat-capacity measurements by adiabatic equilibrium calorimetry are reported for $\gamma$-phase $\operatorname{Pr}_{2} S_{3}, \mathrm{~Tb}_{2} \mathrm{~S}_{3}$, and $\mathrm{Dy}_{2} \mathrm{~S}_{3}$ between 5 and $350 \mathrm{~K}$. Highly purified samples were prepared and their composition verified by chemical analysis. Precision lattice parameters were determined for each compound and are compared with literature values. The total heat capacity has been resolved into lattice, magnetic, and Schottky components by a volumetric approach. The experimental Schottky contributions accord with the calculated curves based on the crystalfield splitting of the ${ }^{2 S+1} L_{J}$ ground state of the lanthanide ions occupying sites of $\mathrm{S}_{4}$ symmetry in the $\mathrm{Th}_{3} \mathrm{P}_{4}$ lattice. The individual crystal-field electronic energy levels have been obtained in part from an analysis of the hot-band data observed in the absorption spectra of $\operatorname{Pr}_{2} \mathrm{~S}_{3}, \mathrm{~Tb}_{2} \mathrm{~S}_{3}$, and $\mathrm{Dy}_{2} \mathrm{~S}_{3}$, and from a calculated splitting in which the crystal-field parameters $B_{k m}$, were determined from a lattice-sum calculation. Molar thermodynamic properties are reported for all three compounds. The entropy at $298.15 \mathrm{~K}\left\{S^{0}-S^{0}(7 \mathrm{~K})\right\}$, is $22.78 R, 22.93 R$, and 23.36R, for $\gamma$-phase $\operatorname{Pr}_{2} \mathrm{~S}_{3}, \mathrm{~Tb}_{2} \mathrm{~S}_{3}$, and $D y_{2} \mathrm{~S}_{3}$, respectively.
\end{abstract}

\section{INTRODUCTION}

In the first paper of this series (referred to as I) we presented the thermophysical properties of four lanthanide sesquisulfides having the $\mathrm{Th}_{3} \mathrm{P}_{4}$ ( $\gamma$-phase) structure, namely $\mathrm{La}_{2} \mathrm{~S}_{3}, \mathrm{Ce}_{2} \mathrm{~S}_{3}, \mathrm{Nd}_{2} \mathrm{~S}_{3}$, and $\mathrm{Gd}_{2} \mathrm{~S}_{3}{ }^{1}$ Interest in these materials arises from their success as components in solar energy absorbers and photovoltaic cells. ${ }^{1-6}$ The defect structure of the material allows one to explore a range of electronic transport phenomena as the solid-solution between $R_{2} S_{3}$ and $R_{3} S_{4}$ involves a change from insulating to semimetallic characteristics without a change in phase. ${ }^{7-14}$

In this paper we extend the analyses given in I to other lanthanide sesquisulfides that can be prepared in the same phase. These compounds include $\gamma$-phase $\operatorname{Pr}_{2} \mathrm{~S}_{3}, \mathrm{~Tb}_{2} \mathrm{~S}_{3}$, and $D y_{2} S_{3}$. The compound $\mathrm{Sm}_{2} \mathrm{~S}_{3}$ is a special case that we reserve for discussion in a later paper. Literature heat-capacity values for $\gamma$-phase $\operatorname{Pr}_{2} \mathrm{~S}_{3}, \mathrm{~Tb}_{2} \mathrm{~S}_{3}$, and $\mathrm{Dy}_{2} \mathrm{~S}_{3}$, do not extend to sufficiently high temperatures to determine the Schottky contribution due to the crystal-field electronic energy levels associated with the ${ }^{2 S+1} L_{J}$ ground-state manifold of the tripositive lanthanide ion. ${ }^{13}$ Moreover, their samples were not well characterized as to their chemical composition.

In this paper we include additional details of the sample preparation, and verify the crystal structure of samples investigated. ${ }^{7}$ We report heat-capacity measurements between 5 and $350 \mathrm{~K}$ resolved into lattice, magnetic, and Schottky components for $\gamma$-phase $\operatorname{Pr}_{2} S_{3}, \mathrm{~Tb}_{2} \mathrm{~S}_{3}$, and $\mathrm{Dy}_{2} \mathrm{~S}_{3}$, based on the crystal-field electronic energy levels of the ground-state manifold of $\operatorname{Pr}^{3+}\left({ }^{3} H_{4}\right), \mathrm{Tb}^{3}+\left({ }^{7} F_{6}\right)$, and $\mathrm{Dy}^{3+}\left({ }^{6} H_{15 / 2}\right)$. A detailed analysis of the crystal structure of the $\gamma$ phase of these sesquisulfides indicates that the lanthanide ions occupy sites of $S_{4}$ symmetry. ${ }^{15-21}$ We conclude that the volumetric method used to determine the Schottky contribution represents a valid approach to the analysis of molar heat-capacity data for all $\gamma$-phase $\operatorname{Ln}_{2} S_{3}$ compounds. ${ }^{1,20,22}$

\section{SAMPLE PREPARATION}

The $\mathrm{Pr}_{2} \mathrm{~S}_{3}, \mathrm{~Tb}_{2} \mathrm{~S}_{3}$, and $D y_{2} \mathrm{~S}_{3}$ samples were prepared by direct combination of the pure elements in a manner similar to that described earlier. ${ }^{23}$ The lanthanide metals were prepared in the Ames Laboratory and had the chemical analyses typical of the highly purified metals produced in that laboratory. ${ }^{24}$ Sublimed sulfur $(99.999 \%)$ was contained from ASARCO. ${ }^{25}$

After the synthesis was carried out in sealed quartz ampoules; the material was not melted as it had been in a previous study. ${ }^{23}$ Instead, the quartz ampoules were opened and the sulfide ground and sieved to 200 mesh powder. The powder was cold pressed into pellets $3 \mathrm{~mm}$ thick by $15 \mathrm{~mm}$ diameter. The pressed pellets of $\operatorname{Pr}_{2} \mathrm{~S}_{3}$ were heated to $1500^{\circ} \mathrm{C}$ under a dynamic $\mathrm{H}_{2} \mathrm{~S}$ atmosphere for $9 \mathrm{~h}$. The $\mathrm{Tb}_{2} \mathrm{~S}_{3}$ and $\mathrm{Dy}_{2} \mathrm{~S}_{3}$ pellets were heated to $1450^{\circ} \mathrm{C}$ for $48 \mathrm{~h}$ under flowing $\mathrm{H}_{2} \mathrm{~S}$.

Debye-Scherrer $\mathrm{x}$-ray patterns taken at $295 \mathrm{~K}$ con- 
TABLE I. Lattice parameters and analyzed compositions of $\gamma$-phase $\mathrm{Pr}_{2} \mathrm{~S}_{3}, \mathrm{~Tb}_{2} \mathrm{~S}_{3}$, and $\mathrm{Dy}_{2} \mathrm{~S}_{3}$.

\begin{tabular}{|c|c|c|c|c|c|c|}
\hline \multirow[b]{2}{*}{ Sesquisulfide } & \multicolumn{2}{|c|}{ Lattice parameter/Å } & \multirow{2}{*}{$\begin{array}{l}\text { Analyzed } \\
\text { mole ratio } \\
\text { S/Ln }\end{array}$} & \multirow{2}{*}{$\begin{array}{c}\text { Sample } \\
\text { mass } \\
\mathrm{m} / \mathrm{g}\end{array}$} & \multirow{2}{*}{$\begin{array}{c}\text { Molar } \\
\text { mass } \\
\mathrm{M} / \mathrm{g} \mathrm{mol}^{-1}\end{array}$} & \multirow[b]{2}{*}{ Color } \\
\hline & Present study & Reference 7 & & & & \\
\hline $\mathrm{Pr}_{2} \mathrm{~S}_{3}$ & $8.5748 \pm 0.0003$ & 8.573 & $1.500 \pm 0.006$ & 27.719 & 378.007 & Dark green \\
\hline $\mathrm{Tb}_{2} \mathrm{~S}_{3}$ & $8.3334 \pm 0.0003$ & 8.333 & $1.496 \pm 0.002$ & 34.093 & 414.043 & Grey green \\
\hline $\operatorname{Dy}_{2} S_{3}$ & $8.3006 \pm 0.0002$ & 8.292 & $1.499 \pm 0.001$ & 53.573 & 421.192 & Yellow green \\
\hline
\end{tabular}

tained only lines of the $\gamma$-phase structure. Precision lattice parameters were determined by measuring the theta values of the doublets in the back reflection and applying a NelsonRiley extrapolation function to the data. Lattice parameters determined in this study are given in Table I along with literature values. ${ }^{7}$ The final composition was determined by chemical analysis. The sesquisulfides were dissolved in 4.8 $M$ hydrochloric acid and the evolved hydrogen sulfide gas was collected in a solution of sodium hydroxide and hydrogen peroxide. The sulfate which formed was determined as barium sulfate. The lanthanide ions in solution were quantitatively analyzed by titrating with EDTA. An additional test, which was the most sensitive to the presence of trace oxysulfide impurities, was to dissolve the sulfide in $6 \mathrm{~N} \mathrm{HCI}$ and observe the clarity of the solutions. A clear solution indicates the absence of oxysulfide. All samples used in this study gave clear solutions on dissolution.

\section{AUTOMATED ADIABATIC CALORIMETRY}

The heat-capacity data were taken in the Mark X calorimetric cryostat, an improved version of the Mark II cryostat previously described (by addition of a guard shield surrounding the adiabatic shield) together with the relevant operating techniques. ${ }^{26}$ The acquisition of heat-capacity data from about 5 to $350 \mathrm{~K}$ was computer assisted..$^{27}$ A goldplated oxygen-free high-conductivity copper calorimeter (laboratory designation W-61) specially equipped with a pair of perforated copper spring-loaded sleeves soldered to the heater-thermometer well to hold the sulfide pellets was employed for all measurements. After loading the calorimeter was evacuated and about $2.0 \mathrm{kPa}$ (at $300 \mathrm{~K}$ ) helium gas added to facilitate thermal equilibration. The calorimeter seal-off tip was closed with Cerroseal (50 mass percent tin and indium) solder. Temperatures were measured on an ASL-7 induction bridge with a Leeds and Northrup platinum-resistance thermometer sensor calibrated by the National Bureau of Standards (now called NIST). All other accurate experimental quantities are referred to standards and calibrations provided by NBS.

\section{CALORIMETRIC MEASUREMENTS}

Table II presents the molar heat capacities for $\gamma$-phase $\mathrm{Pr}_{2} \mathrm{~S}_{3}, \mathrm{~Tb}_{2} \mathrm{~S}_{3}$, and $\mathrm{Dy}_{2} \mathrm{~S}_{3}$. Relevant adjuvant quantities (sample mass, molar masses, lattice constants, etc.) used in computer reduction of the data are given in Table I. The mole used throughout the paper is based on the formula written as $\mathrm{Ln}_{2} \mathrm{~S}_{3}$ and thus involves two moles of lanthanide ( $\mathrm{Ln}$ ) ca- tions. The data, in energy-dimensionless units, are given in chronological sequence so that the temperature increments can usually be deduced from differences in adjacent temperatures. Data were obtained between 5 and $350 \mathrm{~K}$ and are represented in Fig. 1. They are compared with the results for $\mathrm{La}_{2} \mathrm{~S}_{3}$ that has no excess contribution to the "lattice" $C_{p}$. The low temperature detail of Fig. 1 represents a plot of $C_{p}$ vs $T$ for $\operatorname{Pr}_{2} \mathrm{~S}_{3}, \mathrm{~Tb}_{2} \mathrm{~S}_{3}$, and $\mathrm{Dy}_{2} \mathrm{~S}_{3}$ up to $20 \mathrm{~K}$. The open symbols are data obtained in the present study; the solid symbols represent the results reported by Ho et al. ${ }^{13}$ The results for $\mathrm{Dy}_{2} \mathrm{~S}_{3}$ and $\operatorname{Pr}_{2} \mathrm{~S}_{3}$ are in reasonable agreement, whereas the results for $\mathrm{Tb}_{2} \mathrm{~S}_{3}$ are lower than the values reported by Ho et al. ${ }^{13}$ The difference may be due to the difference in stoichiometry between the two $\mathrm{Tb}_{2} \mathrm{~S}_{3}$ samples. Additionally there is a small peak in our data of unknown origin at $12.4 \mathrm{~K}$ without any relevant feature in the susceptibility measurements. ${ }^{28}$

Although no evidence is found for magnetic ordering in $\mathrm{Pr}_{2} \mathrm{~S}_{3}$ above $1 \mathrm{~K}$ neither from magnetic susceptibility ${ }^{28}$ nor from heat-capacity measurements, $\mathrm{Dy}_{2} \mathrm{~S}_{3}$ shows antiferromagnetic ordering around $3 \mathrm{~K}$, and $\mathrm{Tb}_{2} \mathrm{~S}_{3}$ has low lying levels below $10 \mathrm{~K}$. This precludes determination of the Debye characteristic temperature at $0 \mathrm{~K}$. For $\mathrm{Pr}_{2} \mathrm{~S}_{3}$ our data accord well with the values of Ho et al. ${ }^{13}$ which extend to lower temperatures (Fig. 1).

The thermodynamic properties of $\gamma$-phase $\operatorname{Pr}_{2} \mathrm{~S}_{3}, \mathrm{~Tb}_{2} \mathrm{~S}_{3}$, and $D y_{2} S_{3}$ between 7 and $350 \mathrm{~K}$ are summarized in Tables III-V. The accuracy is characterized by $<0.1 \%$ standard deviation above $15 \mathrm{~K}$. Below this temperature, standard deviations of the heat-capacity gradually increase until they attain an uncertainty of about two percent at the lowest temperatures.

\section{DETERMINATION OF SCHOTTKY HEAT-CAPACITY CONTRIBUTIONS}

The presence of Schottky contributions to the heat capacities of $\gamma$-phase $\operatorname{Pr}_{2} S_{3}, T_{2} S_{3}$, and $D_{2} S_{3}$ is evident upon comparison with the $\mathrm{La}_{2} \mathrm{~S}_{3}$ and $\mathrm{Gd}_{2} \mathrm{~S}_{3}$ curves reported in $\mathrm{I}$. The volume-weighted lattice heat capacity approximation technique has been used successfully in several other groups of lanthanide compounds (sesquioxides, halides, trihydroxides, etc.) to deduce the vibrational lattice contribution. ${ }^{29,30}$ Of the $\gamma$-phase lanthanide sesquisulfides, only $\mathrm{La}_{2} \mathrm{~S}_{3}$, has not excess contribution in the heat capacity. After subtraction of the low-temperature magnetic contribution in the $\gamma$-phase $\mathrm{Gd}_{2} \mathrm{~S}_{3}$, its lattice heat capacity was used in I together with that of $\mathrm{La}_{2} \mathrm{~S}_{3}$ to interpolate the values for the other isostructural lanthanide sesquisulfides with this technique. This 
TABLE II. Molar heat capacities of lanthanide sesquisulfides $\left(R=8.3144 \mathrm{~J} \mathrm{~K}^{-1} \mathrm{~mol}^{-1}\right)$.

\begin{tabular}{|c|c|c|c|c|c|c|c|}
\hline$T / \mathrm{K}$ & $C_{p} / R$ & $T / \mathrm{K}$ & $C_{p} / R$ & $T / \mathrm{K}$ & $C_{p} / R$ & $T / \mathrm{K}$ & $C_{p} / R$ \\
\hline \multicolumn{8}{|c|}{ Praseodymium sesquisulfide $\left(\operatorname{Pr}_{2} \mathrm{~S}_{3}\right)$} \\
\hline \multicolumn{2}{|c|}{ Series I } & 25.48 & 2.105 & 75.88 & 7.787 & 211.69 & 14.059 \\
\hline 7.26 & 0.455 & 26.80 & 2.235 & 79.81 & 8.208 & 216.87 & 14.100 \\
\hline 7.62 & 0.471 & 28.08 & 2.377 & 84.24 & 8.679 & 222.05 & 14.185 \\
\hline 8.11 & 0.501 & 29.42 & 2.525 & 89.16 & 9.136 & 227.22 & 14.248 \\
\hline 8.58 & 0.537 & 30.82 & 2.682 & 94.11 & 9.530 & 232.40 & 14.295 \\
\hline 9.05 & 0.576 & 32.29 & 2.853 & 99.09 & 9.905 & 237.58 & 14.322 \\
\hline 9.54 & 0.612 & 33.83 & 3.028 & 104.09 & 10.283 & 242.76 & 14.386 \\
\hline 10.02 & 0.646 & 35.44 & 3.213 & 109.10 & 10.628 & 247.93 & 14.458 \\
\hline 10.51 & 0.684 & 37.14 & 3.404 & 114.14 & 10.966 & 253.12 & 14.501 \\
\hline 10.99 & 0.717 & 38.91 & 3.615 & 119.19 & 11.280 & 258.30 & 14.500 \\
\hline 11.49 & 0.760 & 40.77 & 3.826 & 124.25 & 11.562 & 263.49 & 14.582 \\
\hline 12.05 & 0.804 & 42.73 & 4.047 & 129.33 & 11.821 & 268.66 & 14.574 \\
\hline 12.64 & 0.849 & 44.78 & 4.284 & 134.42 & 12.068 & 273.84 & 14.608 \\
\hline 13.25 & 0.900 & 46.93 & 4.549 & 139.51 & 12.296 & 279.02 & 14.629 \\
\hline 13.89 & 0.952 & 49.19 & 4.801 & 144.62 & 12.524 & 284.20 & 14.656 \\
\hline 14.57 & 1.017 & 51.79 & 5.107 & 149.87 & 12.675 & 289.40 & 14.677 \\
\hline 15.27 & 1.079 & 54.67 & 5.434 & 154.87 & 12.850 & 294.62 & 14.645 \\
\hline 16.01 & 1.145 & & & 160.00 & 13.021 & 299.81 & 14.737 \\
\hline 16.78 & 1.213 & \multicolumn{2}{|c|}{ Series II } & 165.14 & 13.155 & 304.98 & 14.808 \\
\hline 17.59 & 1.289 & 51.40 & 5.057 & 170.28 & 13.300 & 310.15 & 14.801 \\
\hline 18.44 & 1.367 & 54.04 & 5.362 & 175.43 & 13.410 & 315.34 & 14.838 \\
\hline 19.32 & 1.452 & 56.93 & 5.694 & 180.58 & 13.526 & 320.51 & 14.869 \\
\hline 20.25 & 1.544 & 59.83 & 6.033 & 185.81 & 13.632 & 325.68 & 14.902 \\
\hline 21.22 & 1.643 & 62.74 & 6.390 & 190.97 & 13.709 & 330.83 & 14.931 \\
\hline 22.24 & 1.748 & 65.62 & 6.711 & 196.15 & 13.812 & 335.99 & 14.967 \\
\hline 23.30 & 1.864 & 68.75 & 7.035 & 201.32 & 13.918 & 341.16 & 15.020 \\
\hline 24.41 & 1.980 & 72.19 & 7.406 & 206.51 & 13.964 & 346.33 & 15.051 \\
\hline \multicolumn{8}{|c|}{ Terbium sesquisulfide $\left(\mathrm{Tb}_{2} \mathrm{~S}_{3}\right)$} \\
\hline \multicolumn{2}{|c|}{ Series I } & 13.14 & 0.839 & 59.45 & 6.374 & 184.76 & 13.313 \\
\hline 7.10 & 0.678 & 13.40 & 0.852 & 62.35 & 6.720 & 189.91 & 13.426 \\
\hline 7.77 & 0.654 & 13.88 & 0.875 & 65.40 & 7.051 & 195.07 & 13.522 \\
\hline 8.30 & 0.652 & 14.72 & 0.938 & 68.29 & 7.348 & 200.23 & 13.612 \\
\hline 8.83 & 0.656 & 15,67 & 1.010 & 70.85 & 7.585 & 205.40 & 13.668 \\
\hline 9.32 & 0.675 & 16.62 & 1.090 & 74.38 & 7.929 & 210.57 & 13.733 \\
\hline 9.79 & 0.684 & 17.56 & 1.173 & 78.05 & 8.286 & 215.74 & 13.806 \\
\hline 10.28 & 0.708 & 18.51 & 1.264 & 81.92 & 8.659 & 220.91 & 13.874 \\
\hline 10.79 & 0.724 & 19.46 & 1.360 & 85.89 & 9.031 & 226.08 & 13.962 \\
\hline 11.32 & 0.758 & 20.41 & 1.460 & 87.89 & 9.125 & 231.26 & 14.011 \\
\hline 11.88 & 0.792 & 21.38 & 1.569 & 90.44 & 9.399 & 236.44 & 14.066 \\
\hline 12.45 & 0.841 & 22.40 & 1.690 & 95.46 & 9.744 & 241.62 & 14.094 \\
\hline 13.07 & 0.831 & 23.46 & 1.812 & 100.45 & 10.063 & 246.78 & 14.190 \\
\hline 13.73 & 0.864 & 24.57 & 1.955 & 105.46 & 10.387 & 251.96 & 14.229 \\
\hline 14.40 & 0.908 & 25.67 & 2.905 & 110.48 & 10.690 & 257.14 & 14.261 \\
\hline 15.10 & 0.964 & 26.82 & 2.241 & 115.53 & 10.959 & 263.33 & 14.309 \\
\hline 15.83 & 1.022 & 28.09 & 2.407 & 120.60 & 11.241 & 267.51 & 14.325 \\
\hline 16.59 & 1.086 & 29.42 & 2.581 & 125.68 & 11.505 & 272.69 & 14.388 \\
\hline 17.39 & 1.158 & 30.82 & 2.770 & 130.77 & 11.728 & 277.87 & 14.406 \\
\hline 18.23 & 1.235 & 32.29 & 2.964 & 135.86 & 11.944 & 283.06 & 14.468 \\
\hline \multirow[t]{2}{*}{19.10} & 1.323 & 33.82 & 3.171 & 140.97 & 12.121 & 288.24 & 14.493 \\
\hline & & 35.43 & 3.390 & 146.09 & 12.308 & 293.43 & 14.520 \\
\hline \multicolumn{2}{|c|}{ Series II } & 37.12 & 3.614 & 151.21 & 12.478 & 298.61 & 14.535 \\
\hline 10.00 & 0.692 & 38.90 & 3.851 & & & 303.79 & 14.578 \\
\hline 10.51 & 0.717 & 40.76 & 4.088 & \multicolumn{2}{|c|}{ Series III } & 308.98 & 14.616 \\
\hline 11.00 & 0.737 & 42.72 & 4.342 & 149.59 & 12.426 & 314.17 & 16.675 \\
\hline 11.50 & 0.772 & 44.77 & 4.608 & 154.72 & 12.564 & 319.35 & 14.722 \\
\hline 11.88 & 0.789 & 46.93 & 4.879 & 159.86 & 12.703 & 324.55 & 14.753 \\
\hline 12.12 & 0.819 & 49.19 & 5.166 & 165.00 & 12.865 & 329.74 & 14.807 \\
\hline 12.37 & 0.853 & 51.57 & 5.458 & 170.13 & 12.997 & 334.92 & 14.864 \\
\hline 12.62 & 0.846 & 54.07 & 5.753 & 174.44 & 13.103 & 340.11 & 14.900 \\
\hline 12.88 & 0.822 & 56.69 & 6.058 & 179.61 & 13.201 & 345.29 & 14.984 \\
\hline \multicolumn{8}{|c|}{ Dysprosium sesquisulfide $\left(\mathrm{Dy}_{2} \mathrm{~S}_{3}\right)$} \\
\hline \multicolumn{2}{|c|}{ Series I } & 158.75 & 13.334 & 320.29 & 15.095 & 29.44 & 2.347 \\
\hline 61.77 & 6.758 & 163.89 & 13.443 & 325.48 & 15.074 & 30.83 & 2.549 \\
\hline 64.82 & 7.156 & 169.04 & 13.559 & 330.68 & 15.088 & 32.31 & 2.741 \\
\hline 67.99 & 7.523 & 174.18 & 13.690 & 335.86 & 15.141 & 33.84 & 2.964 \\
\hline 71.33 & 7.902 & 179.34 & 13.767 & 341.06 & 15.156 & 35.45 & 3.197 \\
\hline 74.84 & 8.286 & 185.13 & 13.862 & 346.25 & 15.189 & 37.15 & 3.419 \\
\hline 78.53 & 8.683 & 189.15 & 13.943 & & & 38.92 & 3.682 \\
\hline
\end{tabular}


TABLE II. (continued).

\begin{tabular}{|c|c|c|c|c|c|c|c|}
\hline$T / \mathrm{K}$ & $C_{p} / R$ & $T / \mathrm{K}$ & $C_{p} / R$ & $T / \mathrm{K}$ & $C_{p} / R$ & $T / \mathrm{K}$ & $C_{p} / R$ \\
\hline 82.40 & 9.123 & 194.04 & 14.012 & \multicolumn{2}{|c|}{ Series V } & 40.78 & 3.930 \\
\hline 86.48 & 9.532 & 199.43 & 14.074 & 6.59 & 0.517 & 42.74 & 4.206 \\
\hline 90.77 & 9.888 & 204.60 & 14.155 & 6.92 & 0.523 & 44.79 & 4.496 \\
\hline 95.30 & 10.238 & 209.77 & 14.228 & 7.33 & 0.476 & 46.95 & 4.797 \\
\hline 100.07 & 10.582 & 214.94 & 14.279 & 7.72 & 0.475 & 49.21 & 5.092 \\
\hline 105.02 & 10.928 & 220.11 & 14.330 & 8.12 & 0.472 & 51.59 & 5.416 \\
\hline 110.06 & 11.255 & 225.29 & 14.393 & 8.56 & 0.451 & & \\
\hline 115.10 & 11.551 & 230.46 & 14.435 & 9.27 & 0.452 & \multicolumn{2}{|c|}{ Series VII } \\
\hline 120.14 & 11.836 & 235.64 & 14.485 & 10.21 & 0.460 & 5.93 & 0.66 \\
\hline 125.21 & 12.082 & 240.82 & 14.536 & 11.21 & 0.489 & 6.97 & 0.52 \\
\hline 130.30 & 12.288 & 245.99 & 14.605 & 12.16 & 0.525 & 8.18 & 0.459 \\
\hline 135.38 & 12.513 & 251.17 & 14.620 & 13.10 & 0.571 & 9.59 & 0.452 \\
\hline 140.45 & 12.643 & 256.36 & 14.662 & 14.03 & 0.632 & 11.07 & 0.482 \\
\hline \multirow[t]{2}{*}{145.55} & 12.924 & 261.54 & 14.681 & 14.96 & 0.700 & 12.46 & 0.538 \\
\hline & & 266.72 & 14.713 & 15.89 & 0.773 & 13.81 & 0.613 \\
\hline \multicolumn{2}{|c|}{ Series II } & 271.90 & 14.751 & 16.83 & 0.850 & 15.14 & 0.710 \\
\hline 79.86 & 8.821 & 277.09 & 14.780 & 17.76 & 0.938 & 18.21 & 0.976 \\
\hline 82.75 & 9.143 & 282.27 & 14.828 & 18.69 & 1.030 & 19.80 & 1.144 \\
\hline 86.87 & 9.562 & 287.45 & 14.847 & 19.88 & 1.152 & & \\
\hline 91.20 & 9.935 & 292.62 & 14.907 & 21.06 & 1.283 & \multicolumn{2}{|c|}{ Series VIII } \\
\hline 95.75 & 10.275 & 297.80 & 14.951 & 22.05 & 1.400 & 45.62 & 4.608 \\
\hline 100.37 & 10.586 & 302.98 & 14.999 & 23.09 & 1.524 & 47.98 & 4.928 \\
\hline \multirow[t]{2}{*}{105.18} & 10.930 & 308.15 & 15.043 & 24.17 & 1.660 & 50.84 & 5.316 \\
\hline & & & & 25.31 & 1.811 & 53.70 & 5.703 \\
\hline \multicolumn{2}{|c|}{ Series III } & \multicolumn{2}{|c|}{ Series IV } & 26.51 & 1.962 & 58.26 & 6.300 \\
\hline 51.64 & 5.426 & 191.06 & 14.014 & & & & \\
\hline 54.57 & 5.815 & 196.20 & 14.089 & \multicolumn{2}{|c|}{ Series VI } & 62.69 & 6.892 \\
\hline 57.20 & 6.157 & 201.20 & 14.157 & 5.55 & 0.75 & 67.42 & 7.476 \\
\hline 59.99 & 6.531 & 206.21 & 14.219 & 6.53 & 0.55 & 77.04 & 8.556 \\
\hline 62.91 & 6.918 & 211.39 & 14.277 & 7.81 & 0.471 & 81.91 & 9.072 \\
\hline 65.99 & 7.291 & 216.57 & 14.338 & 8.95 & 0.446 & 86.81 & 9.583 \\
\hline 69.22 & 7.666 & 221.75 & 14.390 & 9.98 & 0.460 & 91.75 & 9.999 \\
\hline 72.63 & 8.054 & 226.93 & 14.440 & 10.97 & 0.480 & 96.74 & 10.370 \\
\hline 79.97 & 8.848 & 232.11 & 14.466 & 11.93 & 0.517 & 101.75 & 10.710 \\
\hline 83.92 & 9.266 & 237.30 & 14.504 & 12.88 & 0.560 & 106.77 & 11.066 \\
\hline 88.08 & 9.674 & 242.49 & 14.537 & 13.82 & 0.615 & & \\
\hline 92.47 & 10.031 & 247.67 & 14.572 & 14.75 & 0.682 & & \\
\hline 97.09 & 10.358 & 252.87 & 14.602 & 15.68 & 0.758 & & \\
\hline 101.95 & 10.720 & 258.06 & 14.661 & 16.61 & 0.831 & & \\
\hline 106.05 & 11.049 & 263.24 & 14.711 & 17.54 & 0.916 & & \\
\hline 109.83 & 11.220 & 268.43 & 14.757 & 18.47 & 1.006 & & \\
\hline 112.81 & 11.425 & 273.62 & 14.796 & 19.41 & 1.108 & & \\
\hline 117.89 & 11.727 & 278.80 & 14.824 & 20.35 & 1.203 & & \\
\hline 122.96 & 11.983 & 283.99 & 14.856 & 21.31 & 1.308 & & \\
\hline 128.04 & 12.228 & 289.18 & 14.888 & 22.31 & 1.429 & & \\
\hline 133.13 & 12.462 & 294.37 & 14.933 & 23.36 & 1.577 & & \\
\hline 138.24 & 12.664 & 299.56 & 14.935 & 24.46 & 1.710 & & \\
\hline 143.42 & 12.858 & 304.75 & 15.023 & 25.63 & 1.841 & & \\
\hline 148.50 & 13.007 & 309.93 & 15.002 & 26.84 & 2.007 & & \\
\hline 153.63 & 13.170 & 315.11 & 15.035 & 28.11 & 2.166 & & \\
\hline
\end{tabular}

principle is followed quite accurately in isostructural compounds in which only the cation is changed.

For example, the lattice heat capacity of $\gamma$-phase $\operatorname{Ln}_{2} \mathrm{~S}_{3}$ may be represented as

$$
\begin{aligned}
C_{p}\left(\operatorname{Ln}_{2} \mathrm{~S}_{3}, \text { lattice }\right)= & x C_{p}\left(\mathrm{Gd}_{2} \mathrm{~S}_{3}, \text { lattice }\right) \\
& +(1-x) C_{p}\left(\mathrm{La}_{2} \mathrm{~S}_{3}\right)
\end{aligned}
$$

in which $x$ is the fractional increment in the molar volume $V$ and is given by the expression

$$
\begin{aligned}
x= & \left\{V\left(\operatorname{Ln}_{2} \mathrm{~S}_{3}\right)-V\left(\mathrm{La}_{2} \mathrm{~S}_{3}\right)\right\} / \\
& \left\{V\left(\mathrm{Gd}_{2} \mathrm{~S}_{3}\right)-V\left(\mathrm{La}_{2} \mathrm{~S}_{3}\right)\right\} .
\end{aligned}
$$

The qualification lattice attached to $\mathrm{Gd}_{2} \mathrm{~S}_{3}$ is a reminder that the cooperative magnetic contribution-insignificant above $50 \mathrm{~K}$-has been subtracted. The excess heat capacities (Schottky contributions) have been obtained for $\gamma$-phase $\mathrm{Pr}_{2} \mathrm{~S}_{3}, \mathrm{~Tb}_{2} \mathrm{~S}_{3}$, and $\mathrm{Dy}_{2} \mathrm{~S}_{3}$ after subtracting the calculated lattice contributions and are displayed by continuous curves in Figs. 2-4. 


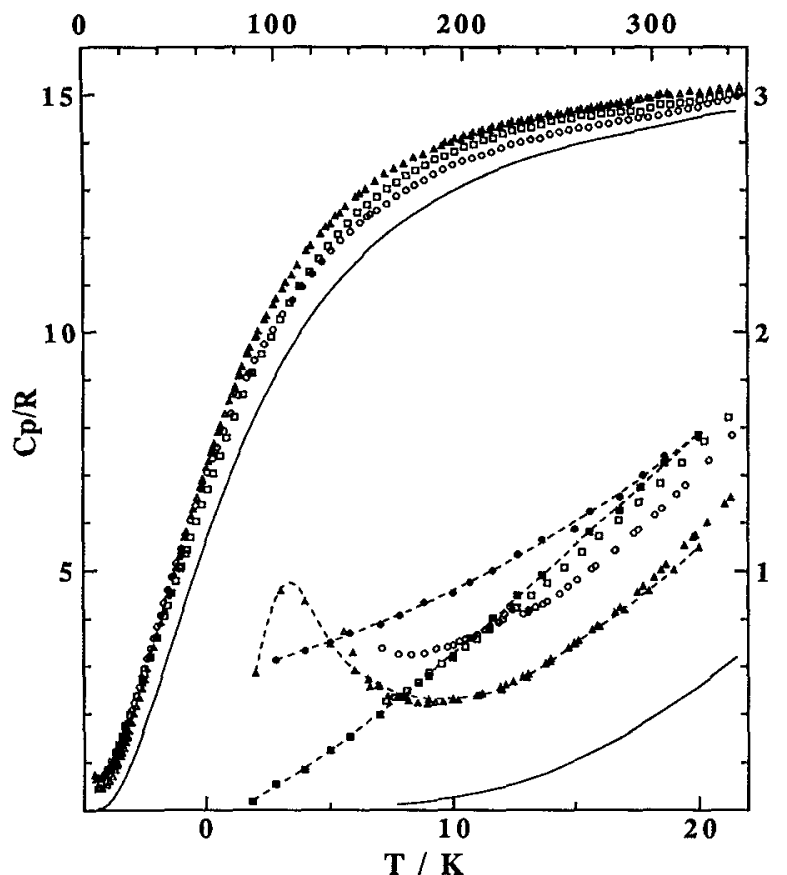

FIG. 1. Molar heat capacities of $\gamma$-phase $\operatorname{Pr}_{2} S_{3},(\square), \mathrm{Tb}_{2} S_{3},(O)$, and $\mathrm{Dy}_{2} \mathrm{~S}_{3},(\Delta)$ from 5 to $350 \mathrm{~K}$. The continuous line represents the "lattice" heat capacity for $\gamma$-phase $\mathrm{La}_{2} \mathrm{~S}_{3}$ (Ref. 1). Lower right corner: Molar heat capacities up to $20 \mathrm{~K}$; open symbols represent present study, solid symbols are taken from Ref. 13.

\section{ANALYSIS OF OPTICAL SPECTRA}

We compared the Schottky heat-capacity curves (Figs. 2-4) with the Schottky contributions based on the experi- mental crystal-field energy levels for $\mathrm{Pr}^{3}+\left({ }^{3} H_{4}\right), \mathrm{Tb}^{3+}\left({ }^{7} F_{6}\right)$, and $\mathrm{Dy}^{3+}\left({ }^{6} H_{15 / 2}\right)$ in the sesquisulfide lattice. The levels were determined from an analysis of the absorption spectra reported earlier for each compound. $2,10,16-19$

The Schottky heat capacity has been developed using the partition function $Z$ for a general system of $n$ energy levels of energies $E_{i}$ and degeneracies $g_{i}$

$$
Z=\sum_{i=0}^{n} g_{i} \exp \left(-E_{i} / R T\right)
$$

and taking $E_{0}=0$, the average energy of such a system is given by

$$
\bar{E}=Z^{-1} \sum_{i=0}^{n} g_{i} E_{i} \exp \left(-E_{i} / R T\right) .
$$

Hence, the electronic heat capacity is given by

$$
\begin{aligned}
C_{\mathrm{Sch}}= & (d \bar{E} / d T) \\
= & \frac{d}{d T}\left(R T^{2} \frac{d \ln Z}{d T}\right) \\
= & Z^{-2} R^{-1} T^{-2}\left\{Z \sum_{i=1}^{n} g_{i} E_{i}^{2} \exp \left(-E_{i} / R T\right)\right. \\
& \left.-\left[\sum_{i=1}^{n} g_{i} E_{i} \exp \left(-E_{i} / R T\right)\right]^{2}\right\} .
\end{aligned}
$$

The crystals used in those measurements were shown to have a stoichiometry and structure similar to the materials used in the present study and reported in Table I. Further details regarding sample preparation, crystal growth, and optical transmission characteristics of those crystals are given in Refs. 31-33.

TABLE III. Molar thermodynamic properties of $\gamma$-phase $\operatorname{Pr}_{2} \mathrm{~S}_{3}\left(R=8.3144 \mathrm{~J} \mathrm{~K}^{-1} \mathrm{~mol}^{-1}\right)$.

\begin{tabular}{ccccc}
\hline$T / \mathrm{K}$ & $C_{p} / R$ & $\left\{S^{0}-S^{0}(7)\right\} / R$ & $\left\{H^{0}-H^{0}(7)\right\} / R \mathrm{~K}$ & $-\left\{G^{0}-G^{0}(7)\right\} / R T$ \\
\hline 7 & 0.499 & 0.0 & 0.0 & 0.0 \\
10 & 0.643 & 0.127 & 1.140 & 0.013 \\
15 & 1.053 & 0.463 & 5.353 & 0.106 \\
20 & 1.521 & 0.828 & 11.764 & 0.240 \\
25 & 2.041 & 1.223 & 20.650 & 0.397 \\
30 & 2.593 & 1.643 & 32.226 & 0.569 \\
40 & 3.735 & 2.545 & 63.84 & 0.949 \\
50 & 4.898 & 3.503 & 107.01 & 1.363 \\
60 & 6.061 & 4.500 & 161.82 & 1.802 \\
70 & 7.175 & 5.518 & 228.06 & 2.260 \\
80 & 8.210 & 6.545 & 305.06 & 2.732 \\
90 & 9.147 & 7.567 & 391.92 & 3.212 \\
100 & 9.978 & 8.575 & 487.64 & 3.698 \\
120 & 11.328 & 10.519 & 701.4 & 4.675 \\
140 & 12.310 & 12.344 & 938.3 & 5.641 \\
160 & 13.009 & 14.036 & 1191.9 & 6.586 \\
180 & 13.510 & 15.60 & 1457.4 & 7.502 \\
200 & 13.879 & 17.04 & 1731.4 & 8.385 \\
220 & 14.158 & 18.38 & 2011.9 & 9.233 \\
240 & 14.368 & 19.62 & 2297.3 & 10.048 \\
260 & 14.526 & 20.78 & 2586.3 & 10.829 \\
280 & 14.645 & 21.86 & 2878.1 & 11.579 \\
298.15 & 14.739 & 22.78 & 3144.7 & 12.233 \\
300 & 14.749 & 22.87 & 3172.0 & 12.298 \\
320 & 14.864 & 23.83 & 3468.1 & 12.989 \\
350 & 15.08 & 25.17 & 3917.2 & 13.976 \\
& & & & \\
\hline \hline
\end{tabular}


TABLE IV. Molar thermodynamic properties of $\gamma$-phase $\mathrm{Tb}_{2} \mathrm{~S}_{3}\left(R=8.3144 \mathrm{~J} \mathrm{~K}^{-1} \mathrm{~mol}^{-1}\right)$.

\begin{tabular}{ccccc}
\hline \hline$T / \mathrm{K}$ & $C_{\rho} / R$ & $\left\{S^{0}-S^{0}(7)\right\} / R$ & $\left\{H^{0}-H^{0}(7)\right\} / R \mathbf{K}$ & $-\left\{G^{0}-G^{0}(7)\right\} / R T$ \\
\hline 7 & 0.693 & 0.000 & 0.0 & 0.0 \\
10 & 0.701 & 0.236 & 1.989 & 0.037 \\
15 & 0.961 & 0.566 & 6.096 & 0.159 \\
20 & 1.418 & 0.900 & 11.955 & 0.302 \\
25 & 2.010 & 1.278 & 20.493 & 0.459 \\
30 & 2.658 & 1.701 & 32.143 & 0.630 \\
40 & 3.991 & 2.650 & 65.43 & 1.014 \\
50 & 5.253 & 3.678 & 111.75 & 1.443 \\
60 & 6.440 & 4.742 & 170.29 & 1.904 \\
70 & 7.519 & 5.817 & 240.18 & 2.386 \\
80 & 8.474 & 6.885 & 320.25 & 2.882 \\
90 & 9.310 & 7.933 & 409.27 & 3.385 \\
100 & 10.035 & 8.952 & 506.1 & 3.891 \\
120 & 11.209 & 10.890 & 719.1 & 4.898 \\
140 & 12.089 & 12.688 & 952.5 & 5.884 \\
160 & 12.745 & 14.347 & 1201.2 & 6.840 \\
180 & 13.228 & 15.88 & 1461.1 & 7.760 \\
200 & 13.587 & 17.29 & 1729.4 & 8.643 \\
220 & 13.865 & 18.60 & 2004.1 & 9.490 \\
240 & 14.093 & 19.82 & 2283.7 & 10.300 \\
260 & 14.283 & 20.95 & 2567.5 & 11.076 \\
280 & 14.435 & 22.02 & 2854.8 & 11.820 \\
298.15 & 14.551 & 22.93 & 3117.8 & 12.469 \\
300 & 14.563 & 23.02 & 3144.8 & 12.533 \\
320 & 14.709 & 23.96 & 3437.4 & 13.218 \\
350 & 15.03 & 25.29 & 3883.4 & 14.197 \\
& & & & \\
\hline \hline
\end{tabular}

Analysis of the crystal structure indicates that the lanthanide ions occupy sites of $S_{4}$ symmetry in the $\gamma$-phase $\mathrm{Ln}_{2} \mathrm{~S}_{3}$ lattice. ${ }^{6,7}$ In $\mathrm{S}_{4}$ symmetry the ground state of $\mathrm{Pr}^{3+}\left({ }^{3} \mathrm{H}_{4}\right)$ is split by the crystalline electric field into five nondegenerate and two doubly degenerate crystal-field electronic energy levels. Since the overall symmetry of the crystal is cubic, it is not possible to carry out the usual polarization experiments to establish the symmetry label for individual levels. However, an analysis of the hot bands made by comparing the absorption spectra observed at 10,80 , and $300 \mathrm{~K}$ establish

TABLE V. Molar thermodynamic properties of $\gamma$-phase $\mathrm{Dy}_{2} \mathrm{~S}_{3}\left(R=8.3144 \mathrm{~J} \mathrm{~K}^{-1} \mathrm{~mol}^{-1}\right)$.

\begin{tabular}{ccccc}
\hline \hline$T / \mathrm{K}$ & $C_{\rho} / R$ & $\left\{S^{0}-S^{0}(7)\right\} / R$ & $\left\{H^{0}-H^{0}(7)\right\} / R \mathrm{~K}$ & $-\left\{G^{0}-G^{0}(7)\right\} / R T$ \\
\hline 7 & 0.510 & 0.0 & 0.0 & 0.0 \\
10 & 0.458 & 0.255 & 1.965 & 0.058 \\
15 & 0.699 & 0.476 & 4.734 & 0.160 \\
20 & 1.167 & 0.737 & 9.331 & 0.271 \\
25 & 1.764 & 1.060 & 16.620 & 0.395 \\
30 & 2.430 & 1.440 & 27.086 & 0.537 \\
40 & 3.826 & 2.330 & 58.35 & 0.871 \\
50 & 5.212 & 3.333 & 103.56 & 1.261 \\
60 & 6.531 & 4.401 & 162.34 & 1.695 \\
70 & 7.760 & 5.501 & 233.88 & 2.160 \\
80 & 8.863 & 6.611 & 317.12 & 2.647 \\
90 & 9.817 & 7.711 & 410.65 & 3.149 \\
100 & 10.619 & 8.790 & 513.0 & 3.659 \\
120 & 11.829 & 10.838 & 738.2 & 4.687 \\
140 & 12.686 & 12.730 & 983.8 & 5.703 \\
160 & 13.338 & 14.468 & 1244.3 & 6.691 \\
180 & 13.827 & 16.07 & 1516.2 & 7.645 \\
200 & 14.155 & 17.54 & 1796.3 & 8.563 \\
220 & 14.360 & 18.90 & 2081.6 & 1.442 \\
240 & 14.518 & 20.16 & 2370.4 & 11.283 \\
260 & 14.680 & 21.33 & 2662.4 & 11.859 \\
280 & 14.828 & 22.42 & 2957.5 & 12.531 \\
298.15 & 14.922 & 23.36 & 3227.5 & 12.598 \\
300 & 14.931 & 23.45 & 3255.1 & 13.307 \\
320 & 15.08 & 24.42 & 3555.0 & 14.318 \\
350 & 15.22 & 25.78 & 4010.2 & \\
& & & & \\
\hline \hline
\end{tabular}




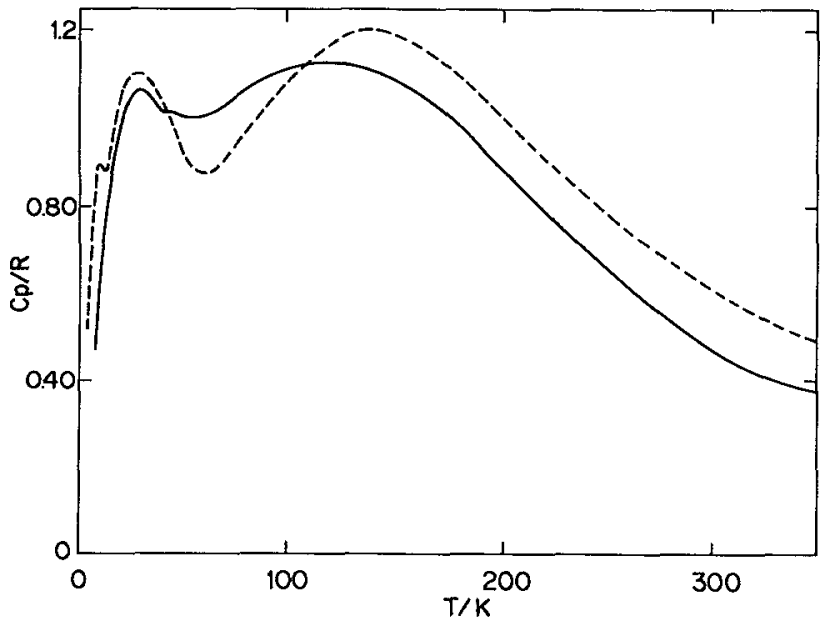

FIG. 2. Schottky contribution to the molar heat capacity of $\gamma$-phase $\mathbf{P}_{2} \mathbf{S}_{3}$; solid line represents calorimetric contribution based on volumetric method; dashed line represents contribution based on crystal-field splitting deduced from the optical spectra.

levels above the ground state level at $12,56,165,265,280$, an $300 \mathrm{~cm}^{-1} \cdot{ }^{10,16}$ The isolated excited states ${ }^{3} P_{0}(20400$ $\mathrm{cm}^{-1}$ ) and ${ }^{3} P_{1}$ (split into two crystal-field electronic energy levels at 20900 and $20950 \mathrm{~cm}^{-1}$ ) provide the best series of hot-bands in the absorption spectra from which we were able to obtain the splitting of the ground state ${ }^{3} H_{4}$. Analysis of the magnetic susceptibility data indicates that the crystal field electronic ground-state level is nondegenerate. ${ }^{16,28}$ In the presence of a magnetic field hot-bands from levels 56 and $265 \mathrm{~cm}^{-1}$ showed pronounced broadening in the absorption spectrum indicating a Zeeman effect due to twofold degeneracy of these levels. ${ }^{10}$ Hot-bands from other electronic energy levels in the ${ }^{3} \mathrm{H}_{4}$ state did not appear to be affected by the magnetic field. Extension of the analysis of the hot-bands in

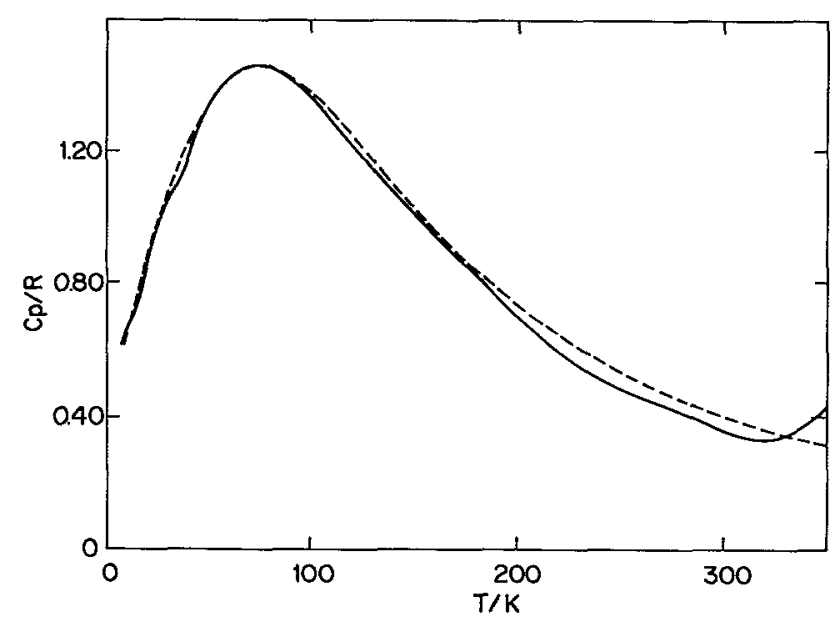

FIG. 3. Schottky contribution to the molar heat capacity of $\gamma$-phase $\mathrm{Tb}_{2} \mathrm{~S}_{3}$; solid line represents calorimetric contribution based on volumetric method; dashed line represents contribution based on crystal-field splitting deduced from the optical spectra.

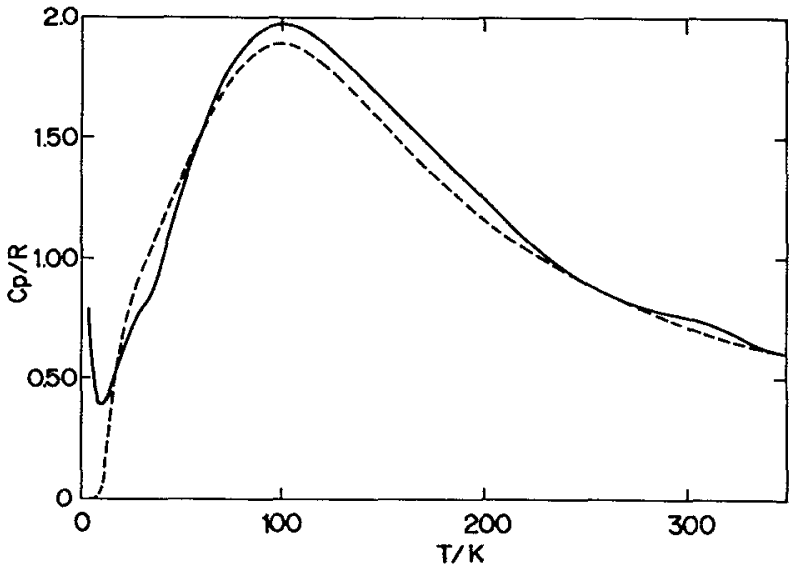

FIG. 4. Schottky contribution to the molar heat capacity of $\gamma$-phase $D y_{2} S_{3}$; solid line represents calorimetric contribution based on volumetric method; dashed line represents contribution based on crystal-field splitting deduced from the optical spectra.

the absorption spectra of ${ }^{3} P_{2}$ and ${ }^{1} D_{2}$ levels await additional experiments to be made on new crystals since the original crystals have deteriorated over the years. A preliminary set of crystal-field electronic energy levels for the ${ }^{3} \mathrm{H}_{4}$ state is given in Table VI. The number in parentheses gives the degeneracy of the level.

The ground state ${ }^{7} F_{6}$ of $\mathrm{Tb}^{3+}$ is split into seven nondegenerate and three doubly degenerate crystal-field electronic energy levels when $\mathrm{Tb}^{3+}$ ions occupy sites of $S_{4}$ symmetry in the sesquisulfide lattice. The excited state ${ }^{5} D_{4}$ contains five nondegenerate and two doubly degenerate crystal-field electronic energy levels. Hot-bands observed in the absorption spectra at 10,80 , and $300 \mathrm{~K}$ of the two lowest levels of this state at 20250 and $20200 \mathrm{~cm}^{-1}$ have been analyzed to give the splitting of the ${ }^{7} F_{6}$ state as follows: $0,6,29,65,130,175$, $190,230,300$, and $310 \mathrm{~cm}^{-1}$. The levels at 6,300 , and 310 $\mathrm{cm}^{-1}$ are estimated by analyzing the shoulders found on several of these hot-bands. ${ }^{16,17}$ Analysis of the magnetic susceptibility data indicates the electronic ground state level and levels at 6 and $29 \mathrm{~cm}^{-1}$ are nondegenerate. ${ }^{16,17}$ The three doubly degenerate levels were chosen on the basis of the prediction made by the lattice-sum calculation given in the next section.

The ground state ${ }^{6} H_{15 / 2}$ of $\mathrm{Dy}^{3+}$ is split into eight doubly degenerate crystal-field electronic energy levels. Part of the absorption spectra has already been published. ${ }^{2,18}$ Subsequent magnetic susceptibility measurements made by Burriel $^{28}$ and the heat-capacity measurements reported in Ref. 4 indicate the $\gamma$-phase $\mathrm{Dy}_{2} \mathrm{~S}_{3}$ undergoes magnetic ordering around $3 \mathrm{~K}$. Analysis of the hot-band absorption spectra at 10,80 , and $300 \mathrm{~K}$ (above the magnetic ordering temperature) establish the splitting of the ${ }^{6} H_{15 / 2}$ as $0,52,145,190$, 240,265 , and $310 \mathrm{~cm}^{-1}$. The lattice sum calculation given in the next section predicts the eighth electronic energy level at $600 \mathrm{~cm}^{-1}$. The room-temperature absorption spectrum contains a hot-band possibly from an electronic level at 550 $\mathrm{cm}^{-1}$. The peak is broad and weak so that the level reported in Table VI must be considered tentative. 
TABLE VI. Crystal-field electronic levels for the ground state, ${ }^{2 S+1} L_{J}$.

\begin{tabular}{|c|c|c|c|c|c|c|c|c|c|c|c|}
\hline $\mathrm{Ln}_{2} \mathrm{~S}_{3}$ & Method of determination & Energy & $\left.m^{-1}\right)$ & nd degen & eracy $(n)$ & of level & & & & & \\
\hline $\operatorname{Pr}_{2} S_{3}$ & Optical spectra/Mag. suscept." & $0(1)$ & $12(1)$ & $56(2)$ & $165(1)$ & $265(2)$ & $280(1)$ & $300(1)$ & & & \\
\hline $\operatorname{Pr}^{3}+\left({ }^{3} H_{4}\right)$ & Lat tice sumb & $O(1)$ & $30(1)$ & $100(2)$ & $180(1)$ & $270(2)$ & $278(1)$ & $282(1)$ & & & \\
\hline $\mathrm{Tb}_{2} \mathrm{~S}_{3}$ & Optical spectra/Mag. suscept.c & $0(1)$ & $6(1)$ & $29(1)$ & $65(2)$ & $130(2)$ & $175(1)$ & $190(1)$ & $230(2)$ & $300(1)$ & $310(1)$ \\
\hline $\mathrm{Tb}^{3}+\left({ }^{7} F_{6}\right)$ & Lattice sumb & $0(1)$ & $5(1)$ & $24(1)$ & $65(2)$ & $130(2)$ & $170(1)$ & $185(1)$ & $235(2)$ & $298(1)$ & $302(1)$ \\
\hline $\mathrm{Dy}_{2} \mathrm{~S}_{\mathrm{i}}$ & Optical spectra/Mag. suscept. ${ }^{d}$ & $O(2)$ & $52(2)$ & $145(2)$ & $190(2)$ & $240(2)$ & $265(2)$ & $310(2)$ & $550(2)$ & & \\
\hline $\mathrm{Dy}^{3}+\left({ }^{6} H_{15 / 2}\right)$ & Lattice sumb & $O(2)$ & $43(2)$ & $140(2)$ & $190(2)$ & $240(2)$ & $280(2)$ & $360(2)$ & $600(2)$ & & \\
\hline
\end{tabular}

References 10 and 16

${ }^{b}$ References $16,17,19$, and 34-36.

'References 16 and 17.

References 2, 18, and 28.

Although the heat-capacity maximum near $3 \mathrm{~K}$ reported by Ho et al. ${ }^{13}$ was interpreted ${ }^{13,18}$ as a consequence of a low-energy electronic level of $\mathrm{Dy}^{3+}$ on the basis of our analysis in this section it must rather be described as due to magnetic ordering. The eight levels of the ground state ${ }^{6} \mathrm{H}_{15 / 2}$ of $\mathrm{Dy}^{3+}$ provide a good fit to the calorimetric Schottky data of Fig. 4. But if one level is used to fit the $3 \mathrm{~K}$ maximum (as an electronic anomaly) the remaining portion of the spectroscopically derived Schottky heat-capacity contribution accounts only for approximately one half of that obtained from calorimetry. This emphasizes the strength of the thermophysical approach.

\section{THEORETICAL SPLITTING OF THE GROUND- STATE MANIFOLD: THE LATTICE SUM APPROACH}

A second approach to establishing the energies of the crystal-field electronic energy levels of the ground state of the trivalent lanthanide ion in the sesquisulfide lattice has been to calculate the theoretical splitting using crystal-field parameters $B_{k m}$ determined from a lattice-sum calculation. Details of this approach are given by Morrison and Leavitt for $\mathrm{Ln}^{3+}$ in an extensive review of the energy levels of lanthanide ions in various host crystals. ${ }^{34-36}$ Specifics related to the sesquisulfide lattice are reported by Gruber, Leavitt, and Morrison. ${ }^{19}$ Following the method of Morrison and Leavitt $^{34}$ a set of $B_{k m}$ parameters for $\mathrm{Ce}^{3+}$ through $\mathrm{Dy}^{3+}$ in $\gamma$ phase $\operatorname{Ln}_{2} \mathrm{~S}_{3}$ was generated and used to interpret magnetic susceptibility data. The results were reported at several conferences. ${ }^{16,17}$ The parameters generated for $\mathrm{Pr}^{3+}, \mathrm{Tb}^{3+}$, and $\mathrm{Dy}^{3+}$ (Refs. 16 and 17) were used in this paper to calculate the splitting of the ${ }^{2 S+1} L_{J}$ ground state for each respective ion.

We present the results of each calculation in Table VI. The number in parentheses represents the predicted degeneracy for the electronic energy level. As we pointed out earlier, the assignment of degeneracy of the levels in the ${ }^{7} F_{6}$ state of $\mathrm{Tb}^{3+}$ is based on the results of the calculation. We were not able to do a Zeeman experiment on the terbium sample due to its poor optical quality. Although only part of the absorption spectra were analyzed, leading us to be cautious regarding these analyses, the results of the theoretical splittings appear to be consistent with our experimental assignments and further suggest that the experimental elec- tronic energy levels deduced from an analysis of the hotbands appearing in the absorption spectra are reliable. The experimental levels can be used to calculate the Schottky contributions to the heat capacity displayed in Figs. 2-4. The overall agreement is reasonable.

In summary, papers I and II for the series of $\gamma$-phase $\mathrm{Ln}_{2} \mathrm{~S}_{3}$ compounds, where $\mathrm{Ln}$ represents $\mathrm{La}$ to $\mathrm{Dy}$, describe the thermophysical properties which can be interpreted using the volumetric method of analysis. The lattice contribution to the heat capacity can be systematically subtracted from the total measured heat capacity leaving the remainder due to the Schottky contribution and some low-temperature magnetic anomalies.

\section{ACKNOWLEDGMENTS}

We wish to thank Professor K. A. Gschneidner, Jr. for many helpful suggestions and comments and Dr. R. Shaviv for help in selecting the Schottky levels to match the calorimetric data. J.B.G. wishes to thank the American Society for Engineering Education for their support during the summer months and Dr. C. A. Morrison, U.S. Army Harry Diamond Laboratories for the program used to calculate the relevant lattice sums and crystal-field splittings determined in this paper. R.B. appreciates financial support from the Comisión Interministerial de Ciencia y Technología, Grant Nos. PPB85-0106 and MAT89-0531. The portion of this research done at the University of Michigan was supported in part by the Structural Chemistry and Chemical Thermodynamics Program of the National Science Foundation under Grant No. CHE-8007977. Sample preparation and characterization carried out at the Ames Laboratory was supported in part by the Office of Basic Energy Sciences, Department of Energy, under Contract No. W-7405-ENG-82. We also wish to thank G. V. Austin and R. Z. Bachman, Analytical Services Group, Ames Laboratory, Iowa State University for determining the composition of the samples used for the heat-capacity measurements.

\footnotetext{
'E. F. Westrum, Jr., R. Burriel, J. B. Gruber, P. E. Palmer, B. J. Beaudry, and W. A. Plautz, J. Chem. Phys. 91, 4838 (1989).

2 J. R. Henderson, M. Muramoto, E. Loh, and J. B. Gruber, J. Chem. Phys. 47, 3347 (1967)

${ }^{3} \mathrm{H}$. J. Goldsmid, in Applications of Thermoelectricity, edited by B. L. Worsnop (Methuen, London, 1960), p. 73.
} 
${ }^{4}$ S. M. A. Taher, J. B. Gruber, J. C. Ho, and D. C. Yeh, in The Rare Earths in Modern Science and Technology, edited by G. J. McCarthy and J. J. Rhyne (Plenum, New York, 1978), Vol. 1, p. 359.

${ }^{5}$ S. M. Taher and J. B. Gruber, Mater. Res. Bull. 16, 1407 (1981).

${ }^{6}$ I. A. Smirnov, J. Phys. (Paris) Suppl. C5 41, 143 (1980).

${ }^{7}$ J. Flahaut, M. Guittard, M. Patrie, M. P. Pardo, S. M. Golabi, and L. Domange, Acta Crystallogr. 19, 14 (1965).

${ }^{8}$ V. P. Zhuze, O. A. Golikova, V. M. Sergeeva, and I. M. Rudick, Sov. Phys. Solid State 13, 669 (1971).

${ }^{9}$ R. M. Bozorth, F. Holtzberg, and S. Methfessel, Phys. Rev. Lett. 14, 952 (1965).

${ }^{10} \mathrm{~J}$. B. Gruber, J. R. Henderson, M. Muramoto, and E. Loh, Bull. Am. Phys. Soc. 14, 310 (1969).

"V. V. Nogteva, O. A. Nabutovskaya, and V. N. Naumov, Russ. J. Phys. Chem. 58, 1573 (1984).

${ }^{12}$ G. Becker, J. Feldhaus, K. Westerholt, and S. Methfessel, J. Magn. Magn. Mat. 6, 14 (1977).

${ }^{13}$ J. C. Ho, S. M. A. Taher, G. B. King, J. B. Gruber, B. J. Beaudry, and K. A. Gschneidner, Jr., J. Phys. (Paris) C6 39, 841 (1978).

${ }^{14}$ K. A. Gschneider, Jr., T. Takeshita, B. J. Beaudry, O. D. McMasters, S. M. A. Taher, J. C. Ho, G. B. King, and J. B. Gruber, J. Phys. (Paris) Suppl. 40, 172 (1979).

${ }^{15}$ H. L. Beeler and J. B. Gruber, Chem. Phys. 13, 359 (1976).

${ }^{16}$ S. M. A. Taher, J. B. Gruber, and B. J. Beaudry, Bull. Am. Phys. Soc. 23, 401 (1978).

${ }^{17}$ S. M. A. Taher, J. C. Ho, and J. B. Gruber, Bull. Am. Phys. Soc. 26, 577 (1981).

${ }^{18}$ S. M. A. Taher, J. C. Ho, and J. B. Gruber, J. Chem. Phys. 76, 609 (1982).

${ }^{19}$ J. B. Gruber, R. P. Leavitt, and C. A. Morrison, J. Chem. Phys. 79, 1664 (1983).

${ }^{20}$ J. B. Gruber, R. Burriel, E. F. Westrum, Jr., P. E. Palmer, and B. J. Beaudry, J. Less-Common Met. 94, 227 (1983).

${ }^{21}$ V. P. Zhuze, M. G. Karin, K. K. Sidorin, V. V. Sokolov, and A. I. Shelykh, Sov. Phys. Solid State 27, 2205 (1986).

${ }^{22}$ E. F. Westrum, Jr., Pure Appl. Chem. 55, 539 (1983).

${ }^{23}$ K. A. Gschneidner, Jr., B. J. Beaudry, T. Takeshita, S. S. Eucker, S. M. A. Taher, J. C. Ho, and J. B. Gruber, Phys. Rev. B 24, 7187 (1981).
${ }^{24}$ B. J. Beaudry and K. A. Gschneidner, Jr., in Handbook on the Physics and Chemistry of Rare Earths, edited by K. A. Gschneidner, Jr. and L. Eyring (North-Holland, Amsterdam, 1978), Vol, 1, p. 173.

${ }^{25}$ American Smelting and Refining Company, Denver.

${ }^{26} \mathrm{E}$. D. West and E. F. Westrum, Jr., in Experimental Themodynamics, edited by J. P. McCullough and D. W. Scott (Butterworths, London, 1968), Vol. 1, p. 333.

${ }^{27}$ E. F. Westrum, Jr., in Thermodynamics and its Applications to Chemical and Biochemical Systems, edited by M. A. V. Ribeiro da Silva (Reidel, Dordrecht, 1984), p. 745.

${ }^{28} \mathrm{R}$. Burriel, Instituto de Ciencia de Materiales de Aragón, Universidad de Zaragoza-CSIC, 50009 Zaragoza, Spain (unpublished).

${ }^{29}$ R. D. Chirico and E. F. Westrum, Jr., J. Chem. Thermodyn. 12, 71, 311 (1980); 13, 519, 1087 (1981).

${ }^{30}$ R. D. Chirico, E. F. Westrum, Jr., J. B. Gruber, and J. Warmkessel, J. Chem. Thermodyn. 11, 835 (1979).

${ }^{31}$ J. R. Henderson, D. M. Johnson, and M. Muramoto, "Production of High Purity Rare Earth Sulfides," U. S. Patent No. 3, 748, 095, issued July 24, 1973, owned by McDonnell-Douglas Corp., Long Beach, CA 90846.

${ }^{32}$ J. R. Henderson, M. Muramoto, D. M. Johnson, and E. Loh, "Purification and Growth of Rare Earth Sesquisulfide Semiconductor Crystals," Douglas Paper 4415, Douglas Aircraft Company, Santa Monica, CA, IRAD Program 88001-700 (1967).

${ }^{33}$ J. R. Henderson, J. B. Gruber, D. M. Johnson, and M. Muramoto, "McDonnell-Douglas Corporation Research on Rare Earth Materials, III. Rare Earth Semiconductors, Heterojunctions of GaAs- $\mathrm{Ln}_{2} \mathrm{~S}_{3}$, Infrared Quantum Counters." McDonnell-Douglas Corp. Yearly Report for 1969.

${ }^{34}$ C. A. Morrison and R. P. Leavitt, in Handbook on the Physics and Chemistry of Rare Earths, edited by K. A. Gschneidner, Jr. and L. Eyring (North-Holland, New York, 1982), Vol. 5, p. 461.

${ }^{35}$ C. A. Morrison, D. E. Wortman, and N. Karayianis, J. Phys. C 9, 191 (1976).

${ }^{36} \mathrm{C}$. A. Morrison, in Lecture Notes in Chemistry, edited by G. Berthier (Springer, New York, 1988), Vol. 47, p. 119. 